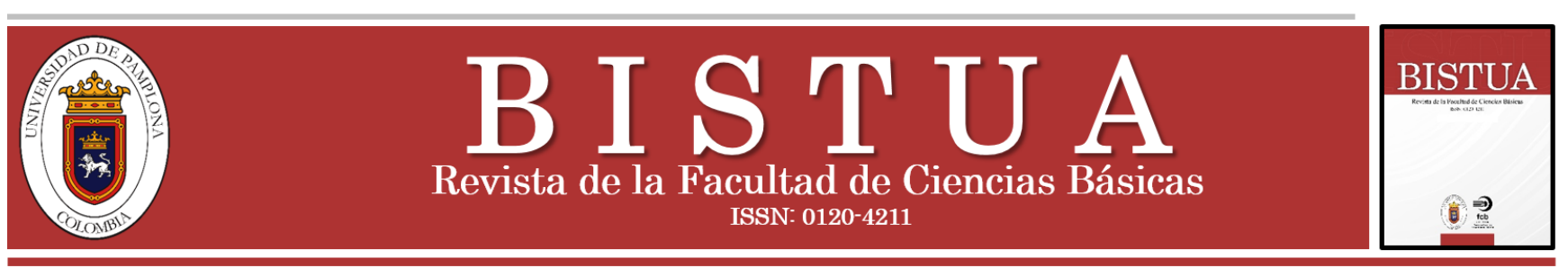

\title{
Estudio de la calidad del aceite Rio de Oro por su índice de refracción asociado con varias frituras
}

\author{
Study of the quality of the Rio de Oro oil by the refractive index associated with various frying \\ Wilmer A. Gómez Fierro; Emiro S. Arrieta-Jiménez \\ ${ }^{a}$ Facultad de Educación, Universidad Surcolombiana, Grupo de Física Teórica, Huila-Colombia. \\ Contactos: u20161146082@usco.edu.co; emiro.arrieta@usco.edu.co
}

Recibido: Febrero 01, 2020. Aceptado: Abril 18, 2020

https://doi.org/10.24054/01204211.v1.n1.2020.4171

\section{Resumen}

Es bien sabido que la mayoría (no todos) de los aceites vegetales, utilizados con frecuencia para cocinar / freír, son beneficiosos para la salud humana. Por ello, es importante usar aceites de buena calidad porque su uso excesivo y / o mala calidad puede desencadenar enfermedades cardiovasculares, diabetes e incluso cáncer. El problema es que, en Colombia, el uso de grasas trans y saturadas está aumentando. En esta contribución se presenta un estudio experimental sobre la calidad del aceite Rio de Oro que se usa ampliamente en Colombia por su bajo costo, para ello se estima el índice de refracción del aceite después de varias frituras, utilizando la expresión del prisma dispersivo integrado con un Espectrómetro-goniómetro. $\mathrm{Al}$ registrar el ángulo mínimo de desviación del espectro de dispersión de la luz blanca utilizada, y usando la fórmula del prisma, se evidencia un aumento en el índice de refracción del aceite mencionado anteriormente, al aumentar el número de fritura hasta 5 veces. Lo que indica que el aceite de Río de Oro es rico en ácidos grasos saturados que son responsables del aumento de la densidad del aceite

Palabras clave: Aceite Rio de Oro; índice de refracción; expresión del prisma dispersivo; freír; espectrómetro-goniómetro.

\section{Introducción}

Los aceites están hechos de nutrientes con diferente composición de ácidos grasos [1]. Estos son importantes no solo por su alto valor energético, sino también por las vitaminas liposolubles que contienen [2]. Estas vitaminas son muy beneficiosas para la salud humana y se encuentran en los alimentos o en los aceites que se utilizan para prepararlas.

Estos ácidos grasos incluyen los saturados y los trans que están relacionados con el colesterol total y el aumento del colesterol LDL [3] lo que, a su vez, aumenta el riesgo de padecer enfermedades cardiovasculares [4] y también ciertos tipos de cáncer [5].

Es por esto que muchos países han desarrollado políticas cuyo objetivo es disminuir la prevalencia de enfermedades

\section{Abstract}

It is well known, that most (not all) vegetable oils, frequently used for cooking/frying purposes, are beneficial for human health. This is why it is important to use good quality oils because their excessive use and/or poor quality may trigger cardiovascular disease, diabetes, and even cancer. The problem is that in Colombia, the use of trans and saturated fat is increasing. This contribution presents an experimental study on the quality of Rio de Oro oil that is widely used in Colombia due to its low cost, for this the refractive index of the oil is estimated after frying, using the dispersive prism method integrated with a Spectrometer-goniometer. By recording the minimum angle of deviation of the scattering spectrum of the white light used, and using the prism formula, an increase in the refractive index of the oil mentioned above is evidenced, by increasing the number of frying up to 5 times. Indicating that the Rio de Oro oil is rich in saturated fatty acids that are responsible for the oil density increase.

Keywords: Rio de Oro oil; refractive index; expression of the dispersive prism; deep-frying; Spectrometer-goniometer.

relacionadas con el consumo de aceites vegetales. En Colombia, la Resolución 2154 de 2012 presenta los requisitos sanitarios, composición y propiedades fisicoquímicas que deben cumplir los aceites y grasas animales y vegetales para ser comercializados en el país, entre ellos indica que sus índice de refracción oscilan entre 1.458 y 1.460 para los aceites de oleína de palma, y para los de soya se encuentra entre 1.466 y 1.470 [6]. El fundamento de esta Resolución fue que, según datos reportados por el Instituto de Evaluación y Medición de la Salud, la enfermedad coronaria isquémica sigue siendo la principal causa de mortalidad en Colombia con un aumento del $21,26 \%$ entre 2005 y 2016 [7].

Esto significa que el estudio de los aceites en general, pero específicamente el de los aceites de cocina, es de suma importancia porque las características de los alimentos fritos 
están estrechamente relacionadas con las propiedades fisicoquímicas y funcionales de los componentes alimentarios [8]. De hecho, investigaciones anteriores han demostrado que existe una estrecha relación entre el deterioro del aceite y los alimentos fritos [9-11]. En la misma línea, el calentamiento sucesivo del aceite también altera la composición química, lo que, a su vez, afecta negativamente la salud humana $[3,12]$.

Hasta donde sabemos, no se ha realizado ninguna investigación sobre la calidad de los aceites en relación con su índice de refracción, siendo este índice un rasgo característico de los aceites [13]. Por lo tanto, es importante poder determinar la calidad del aceite vegetal Río del Oro que, por su bajo costo, es hoy en día el más utilizado para freír en Colombia.

\subsection{Prisma dispersivo}

Cuando un rayo atraviesa un prisma dispersivo de ángulo de ápice $\Phi$, como lo ilustra la Figura 1, saldrá desviado de su orientación original con un ángulo denominado desviación angular $\delta$. Ahora, si el rayo refractado en la primera interfaz es paralelo a su base, se tiene su desviación mínima ( $\delta m i n)$, que al aplicar la ley de Snell en las dos interfaz del prisma, se obtiene el índice de refracción del material que contiene el prisma, dado por $[14,21]$

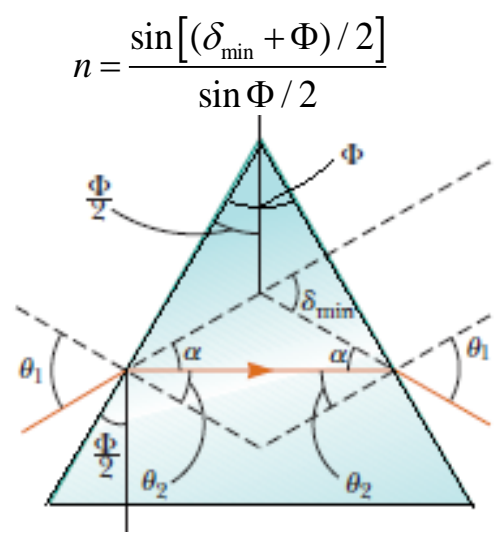

Figura 1. Rayo de luz que pasa a través de un prisma con el angulo de desviacion minimo( $\delta \mathrm{min})$. Fuente: [21].

\section{Método}

\subsection{Diseño}

En la presente investigación se usa un diseño experimental mixto (ver Fig..2) para evaluar la calidad del aceite de Río de Oro. Se seleccionaron las condiciones más frecuentemente utilizadas en las que se fríen las petaconas, con sus valores máximo y mínimo: temperatura $\left(140-200{ }^{\circ} \mathrm{C}\right)$, período de tiempo $(10$ - $15 \mathrm{~min})$ y cantidad de aceite $(1 \mathrm{~L})$ correspondiente a 100 gr de plátano verde / verde.

\subsection{Proceso de fritura}

El alimento frito elegido para este estudio fue el plátano verde / maduro, llamado hartón (Musa paradisiaca). Se pela y se corta en 10 rodajas simétricas. Las 10 rodajas se pusieron en una sartén que contenía aceite a una temperatura media de $140-200{ }^{\circ} \mathrm{C}$ durante 8 minutos hasta que se doraron. Se tomó una muestra de aceite después de 8 minutos de fritura cuando las rodajas se tornaron de color marrón dorado, lo que indica el estado frito. A continuación, se sacaron las 10 rodajas de la sartén y se aplanaron firmemente para preparar las petaconas. Luego se volvieron a poner a freír en el mismo aceite, 2 a la vez (así teníamos 5 lotes de petaconas). Cuando estuvo listo cada lote de 2 petaconas, se extrajeron del recipiente $5 \mathrm{ml}$ de aceite y se analizaron. En total, entonces, teníamos 6 muestras de aceite: 1 del aceite en su forma pura / no frita, y 5 de cada lote de rodajas de plátano frito.

\subsection{Determinación del índice de refracción}

En esta parte se utilizó un prisma hueco (altura: $2,2 \mathrm{~cm}$, ancho 3,3 cm), el cual, se llenó con las 6 muestras de aceite de Río de Oro tomadas previamente. El prisma se colocó en un espectrómetro-goniómetro (Fig. 2). Se proyectó un rayo de luz blanca en la primera interfaz de tal manera que se propagó en paralelo a la base.

La desviación angular mínima del haz rojo refractado por el prisma se determinó utilizando el goniómetro cuya precisión es de 1 minuto. Este valor se insertó en la Ec.(1), lo que indica el índice de refracción de cada muestra de aceite:

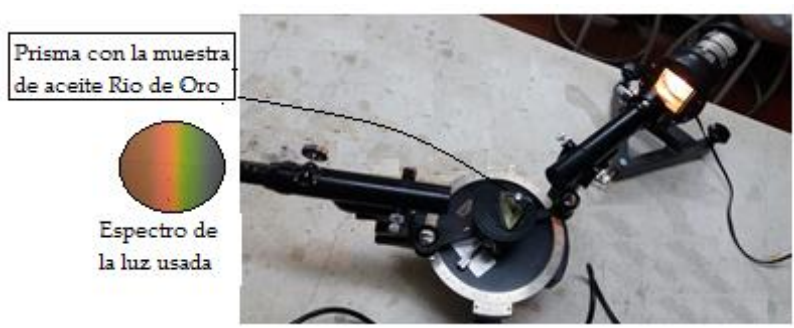

Figura 2. Montaje experimental para la detección del índice de refracción de las muestras de aceite Río de Oro mediante espectrómetrogoniómetro. Fuente: Autor(es)

\section{Resultados y discusión}

En la Tabla 1, se muestran los datos experimentales del índice de refracción medio del aceite puro (es decir, no frito) de Río de Oro $(\overline{\boldsymbol{n}})$ así como su respectivo espectro de dispersión y una estimación del error cometido (dn) para cada medida. 
Tabla 1. Mediciones experimentales de índice de refracción del aceite Rio de Oro con $\Phi=60^{\circ}$.

\begin{tabular}{|c|c|c|c|c|}
\hline $\mathbf{N}^{\circ}$ & $\delta \min \left({ }^{\circ}\right)$ & $\mathrm{n}$ & $\bar{n}$ & $d n$ \\
\hline 1 & 33.375 & 1.45525 & \multirow{2}{*}{1.45029} & 0.00017 \\
\hline 2 & 33.55 & 1.44533 & & 0.00017 \\
\hline
\end{tabular}

Según la literatura, el aceite de Río de oro no es un aceite puro [15]. De hecho, es una mezcla de oleína de soja y palma, que contiene Terbutil Hydroquinone (TBHQ) como antioxidante. Además, hasta donde sabemos, la literatura no menciona el índice de refracción del aceite de Río de Oro, ya que, ese índice (mencionado en la Resolución 2154 [6]) solo se refiere a los aceites puros. Por lo tanto, no se puede hacer una comparación directa del índice de refracción del aceite de Río de Oro y el de los aceites puros. Sin embargo, se puede esperar que su índice de refracción se encuentre entre de los resultados de valores máximos y mínimos que se presentan en esta Resolución2154, tal como lo indica los resultados experimentales de la Tabla 1.

Las propiedad física de los aceites vegetales permite inferir cuál es su composición, porque investigaciones previas han demostrado que existe una relación directa entre, por un lado, el índice de refracción del aceite y su nivel de saturación, y, por otro, el índice de refracción de los aceites y los dobles enlaces cis / trans [16]. Los experimentos realizados con el aceite de Río de oro revelaron una alta proporción de ácidos grasos. La oleína de palma (la fracción del aceite de palma rica en ácido oleico) es el componente responsable de la saturación del aceite porque es rica en ácidos grasos saturados (ácido palmítico) [17]. Es bien sabido que estos ácidos son perjudiciales para la salud humana.

A continuación, se muestra el espectro de dispersión de la luz utilizada cuando atraviesa el prisma con la muestra de aceite de Río de oro (Figura 3).

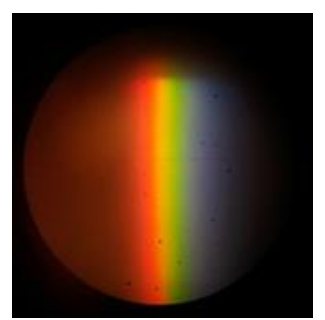

Figura 3. Espectro de dispersión de la luz utilizada al pasar por la muestra de aceite de Río de Oro. Fuente: Autor(es)

En el espectro de dispersión mostrado en la Fig.3, se evidencia que las líneas espectrales no están bien definidas, lo que indica que este aceite presenta bajo poder de resolución. Esto se debe a que la rapidez de la luz varia conforme a la densidad del medio, y este a su vez de la refracción de sus longitudes de ondas, por lo tanto, entre más denso sea el aceite, menor va a ser la resolución de su espectro $[18,19]$.

\section{1 Índice de refracción del aceite según el número de frituras}

En los siguientes datos experimentales mostrados en la Tabla 2 , indican el índice de refracción medio $(\overline{\boldsymbol{n}})$ y el error por central del aceite de Río de Oro después de 5 frituras sucesivas de las rodajas de plátano verde con respecto a su valor hallado sin usar.

\begin{tabular}{ccccc}
\multicolumn{6}{c}{ Tabla 2. Mediciones experimentales del aceite Rio de Oro con $\Phi=60^{\circ}}$. \\
$\begin{array}{c}\text { N } \\
\text { Frituras }\end{array}$ & medición 1 & medición 2 & $\overline{\boldsymbol{n}}$ & $\% \mathrm{E}$ \\
\hline 1 & 1.45865 & 1.45722 & 1,45794 & 0.5 \\
2 & 1.46419 & 1.45820 & 1,46125 & 0.8 \\
3 & 1.46865 & 1.46882 & 1,46873 & 1,3 \\
4 & 1.47060 & 1.46633 & 1,46847 & 1.3 \\
5 & 1.47184 & 1.47361 & 1,47273 & 1.6 \\
\hline
\end{tabular}

Los datos mostrados en la Tabla 2 revelan un aumento importante en el índice de refracción medio del aceite a medida que aumenta el número de futura de él con petaconas, lo cual, nos muestra bajo la definición clásica del índice de refracción(índice de refracción inversamente proporcional a la velocidad de la luz que atraviesa el material) que la densidad del de este aceite Rio de Oro se va incrementando poco a poco, y se vuelve más notorio a partir de la tercera fritura, tal como lo confirma el error relativo presentado en ella, además estos valores están en concordancia con lo mostrado en la Ref. [6]. En otras palabras, las propiedades fisicoquímicas del aceite se alteran cada vez más a medida que aumenta el número de frituras. Este resultado concuerda con investigaciones previas que evidenciaron una degradación del aceite relacionada con el número de veces que se fríen las rodajas de plátano y, en consecuencia, con el aumento de temperatura [10].

El resultado anterior se ilustra con las muestras de aceite que se observan a continuación.

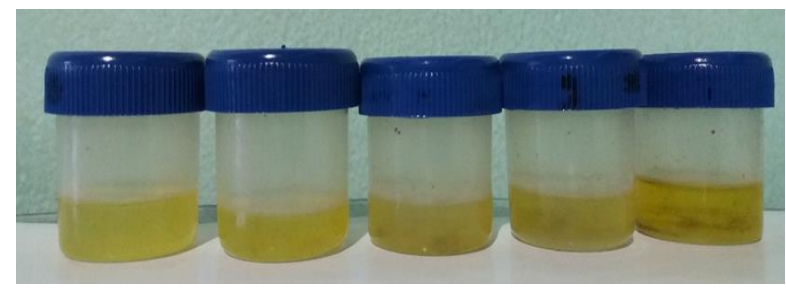

Figura 4. Variación de color respecto a las frituras con plátano del aceite Río de Oro. De izquierda a derecha comienza con la fritura 1. Fuente: Autor(es)

La Figura 4 muestra que las propiedades organolépticas del aceite han cambiado. De hecho, se puede ver que la opacidad del color aumenta con el número de petaconas fritas. Esto se puede observar a partir de la tercera fritura. En la misma línea, los residuos de plátano frito en aceite aumentaron con el número de frituras, lo que provocó un 
cambio en el índice de refracción del aceite porque producen un oscurecimiento prematuro del aceite y una formación excesiva de humo a la temperatura de fritura [20].

También es importante mencionar que este cambio de color podría indicar el nivel de oxidación del aceite. Por tanto, el aceite de Rio de Oro tiene un alto nivel de estabilidad. Este hallazgo está de acuerdo con investigaciones previas que demostraron que el aceite de oleína de palma es más resistente al cambio de color [11].

\section{Conclusiones}

Los resultados de este estudio experimental llevan a la conclusión de que el valor del índice de refracción asociado a la muestra de aceite Río de Oro sin usar, se encuentra entre los valores máximos y mínimos del aceite de oleína de palma que se muestran en la Resolución 2154 de 2012[6], lo cual, confirma una notoria densidad en este aceite, además, indicaría que puede ser rico en ácidos grasos saturados (ácido palmítico), tal como lo anota la Ref.[17].

De manera similar, nuestros hallazgos indican que el índice de refracción aumenta significativamente con la cantidad de veces que se usa el aceite para freír plátanos (ver Tabla 2). Es importante mencionar que esta característica podría ser perjudicial para la salud del ser humano, ya que se convertiría en un factor a tener en cuenta para el aumento del colesterol LDL. En consecuencia, conforme a estos resultados obtenidos se recomienda no utilizar el aceite de Río de Oro por más de 3 frituras, ya que, a partir de ésta se observar un notorio aumento de su densidad que puede ser perjudicial para la salud de las personas, así mismo evitar la formación de desechos sólidos que se sabe que aceleran el proceso de oxidación.

\section{Referencias}

[1] Carrillo Fernández L. C., Dalmau Serra J., Martínez J. R., Solà Alberich A., y Pérez Jiménez F. Dietary fats and cardiovascular health. Aten. Primaria. 43(3) (2011) 1-157. doi: 10.1016/j.aprim.2010.12.003

[2] Murray R. K., Bender D. A., Botham K. M., Kennelly P. J., Rodwell V. W., Weil P. Harper, Bioquímica Ilustrada. $29^{\mathrm{a}}$ ed. México, D.F.: McGraw-Hill, (2013)

[3] Ballesteros Vásquez M. N., Valenzuela Calvillo L. S., Artalejo Ochoa E., Robles Sardin A. E. Ácidos grasos trans: un análisis del efecto de su consumo en la salud humana, regulación del contenido en alimentos y alternativas para disminuirlos. Nutrición Hospitalaria, 27(1) (2012) 54-64. doi:10.3305/nh.2012.27.1.5420

[4] Brotons Cuixart C., Alemán Sánchez J.J., Banegas Banegas J.R., Fondón León C., Lobos Bejarano J.M., Martín Rioboó E., Navarro Pérez J., Orozco Beltrán D., Villar Álvarez F. Recomendaciones preventivas cardiovasculares. Actualización PAPPS 2018. Aten. Primaria. 50(1) (2018) 4-28. doi: 10.1016/S0212-6567(18)30360-3
[5] Kohlmeier L., Simonsen N., van't Veer P., Strain J.J., Martin Moreno J.M., Margolin B., Huttunen J.K., et al. Adipose tissue trans fatty acids and breast cancer in the European Community Multicenter Study on Antioxidants, Myocardial Infarction, and Breast Cancer. Cancer Epidemiology Biomarkers \& Prevention; 6 (1997) 705-710.

[6] Ministerio de salud y protección social. Resolución 2154. República de Colombia. Diario Oficial No. 48.516 (8 de agosto de 2012).

[7] Instituto de Métricas y Evaluación de la Salud. Datos de salud para Colombia. Seattle, Estados Unidos. (2016). http://www.healthdata.org/colombia

[8] Alvis A., Cortés L. E., Páez M. Transferencia de Calor y Materia durante la Fritura de Trozos de Name (Dioscórea alata). Información tecnológica, 20(1) (2009) 99-109. https://dx.doi.org/10.4067/S071807642009000100012

[9] Astudillo Rubio, G. C. Evaluación del deterioro del aceite vegetal en la preparación de papa fritas. (Tesis de Maestría). Universidad del Azuay, Cueca-Ecuador. 2018

[10] Ávila Ávila E. F., Ordóñez Domínguez K. G. Evaluación del grado de degradación primaria del aceite vegetal usado en el proceso de preparación de papas fritas mediante la medición de la formación de peróxidos. (Tesis de grado). Universidad de Cuenca, CuencaEcuador. 2017

[11] Fernández Cedi L. N., Álvarez de la Cadena y Yañez L.b; y Sosa Morales, M. E. Deterioro del aceite de soya y oleína de palma durante el freído de papas a la francesa. XII congreso nacional de ciencia y tecnología de alimentos. 2010. pp. 341-350.

[12] Abilés J., Ramón A. N., Moratalla G., Pérez Abud R., Morón Jiménez J., Ayala A. Efectos del consumo de aceites termo-oxidados sobre la peroxidación lipídica en animales de laboratorio. Nutrición Hospitalaria, 24(4) (2009) 473-478 http://scielo.isciii.es/scielo.php?script=sci_arttext\&pid=S0212$16112009000400012 \& \operatorname{lng}=\mathrm{es} \& \ln \mathrm{n}=\mathrm{es}$

[13] Karabulut, I.; Kayahan, M.; Yaprak, S. Determination of changes in some physical and chemical properties of soybean oil during hydrogenation. Food Chemistry. $812003 \quad 453-456$. doi: https://doi.org/10.1016/S0308-8146(02)00397-7

[14] Hecht, E y Zajac, A. Óptica. Addisson-Wesley Iberoamericana, S.A. Wilmington, Delaware. 1986

[15] Duquesa S.A. Rio de Oro Aceite vegetal. 2014. http://www.duquesa.com.co/cocina_y_mesa/rio_de_oro.html

[16] Delgado Ospina J., Grande Tovar C. D., Menjívar Flores J. C., Sánchez Orozco M. S. Relationship between refractive index and thymol concentration in essential oils of Lippia origanoides Kunth. Chilean journal of agricultural \& animal sciences, 32(2) (2016) 127133. https://dx.doi.org/10.4067/S0719-38902016000200006

[17] Sundram K. Modulation of human lipids and lipoproteins by dietary palm oil and palm olein: a review. Asia Pacific journal of clinical $\begin{array}{llll}\text { nutrition. } & 6(1) & \text { (1997) }\end{array}$ https://pubmed.ncbi.nlm.nih.gov/24394646/

[18] Sears, Z., Young, F. Fisica Universitaria, Vol. II, Pearson, 1999.

[19] Chao Mujica, F. J. Método teórico para la predicción del índice de refracción en refractometría. Boletín Científico Técnico INIMET, (2), (2013) 1-18.

https://www.redalyc.org/articulo.oa?id=2230/223029286002

[20] Viera Guerrero J. P. Estabilidad del aceite de fritura de chifles. (Trabajo de grado). Universidad de Piura, Piura- Perú. 2005 